\title{
An Innovative Casting Technique for Off-Loading Charcot Foot
}

\section{Keywords: Charcot; Off-loading; Total contact; Plaster cast}

\section{Abstract}

Numerous techniques have been described in the use of offloading cast or brace in Charcot osteoarthropathy of the foot and ankle. The dominating challenge remains the inability to adequately conform to the varying shapes of deformities and soft tissue defects. We present a simple modification aimed at improving the existing technique.

\section{Introduction}

The complex syndrome of Charcot foot osteoarthropathy often presents when the cycle of bone destruction and deformity has already begun [1]. Neuropathic arthropathy in the foot secondary to diabetes mellitus remains a major problem with significant health and socioeconomic impact [2]. There are currently no cures for this condition and the mainstay of management consists of prevention and treatment of complications. These options range from preventive medical treatment to corrective surgical procedures. Treatment with cast off-loading to relieve plantar pressure has been shown to reduce ulcer recurrence and progression [1]. We present a simple modification aimed at improving the existing technique.

\section{Journal of \\ Orthopedics \& Rheumatology}

\section{Tarek Boutefnouchet ${ }^{1,2 *}$, Thomas Laios ${ }^{2}$ and Tracey Westwood ${ }^{1}$ \\ ${ }^{I}$ Department of Trauma and Orthopaedics, University Hospital,} Birmingham, UK

${ }^{2}$ Department of Trauma and Orthopaedic Surgery, Heart of England NHS Foundation Trust, Bordesley Green East, Birmingham B9 5SS, UK

*Address for Correspondence

Tarek Boutefnouchet, MBChB, MRCS, PGCert ME, Department of Trauma and Orthopaedics, University Hospital, Mindelsohn Way, Birmingham - B15 2WB, UK, E-mail: tboutefnouchet@hotmail.com

Submission: 29 September, 2016

Accepted: 05 November, 2016

Published: 11 November, 2016

Copyright: ๑ 2016 Boutefnouchet T, et al. This is an open access article distributed under the Creative Commons Attribution License, which permits unrestricted use, distribution, and reproduction in any medium, provided the original work is properly cited.

\section{Technique}

Similar to most institutions patients are selected following a multidisciplinary approach. The aim of this treatment is to protect the architecture of the foot while enhancing the ability of ulcers to heal and preventing further ulceration. A diligent diabetes treatment regime and control of blood glucose is central to the success of total

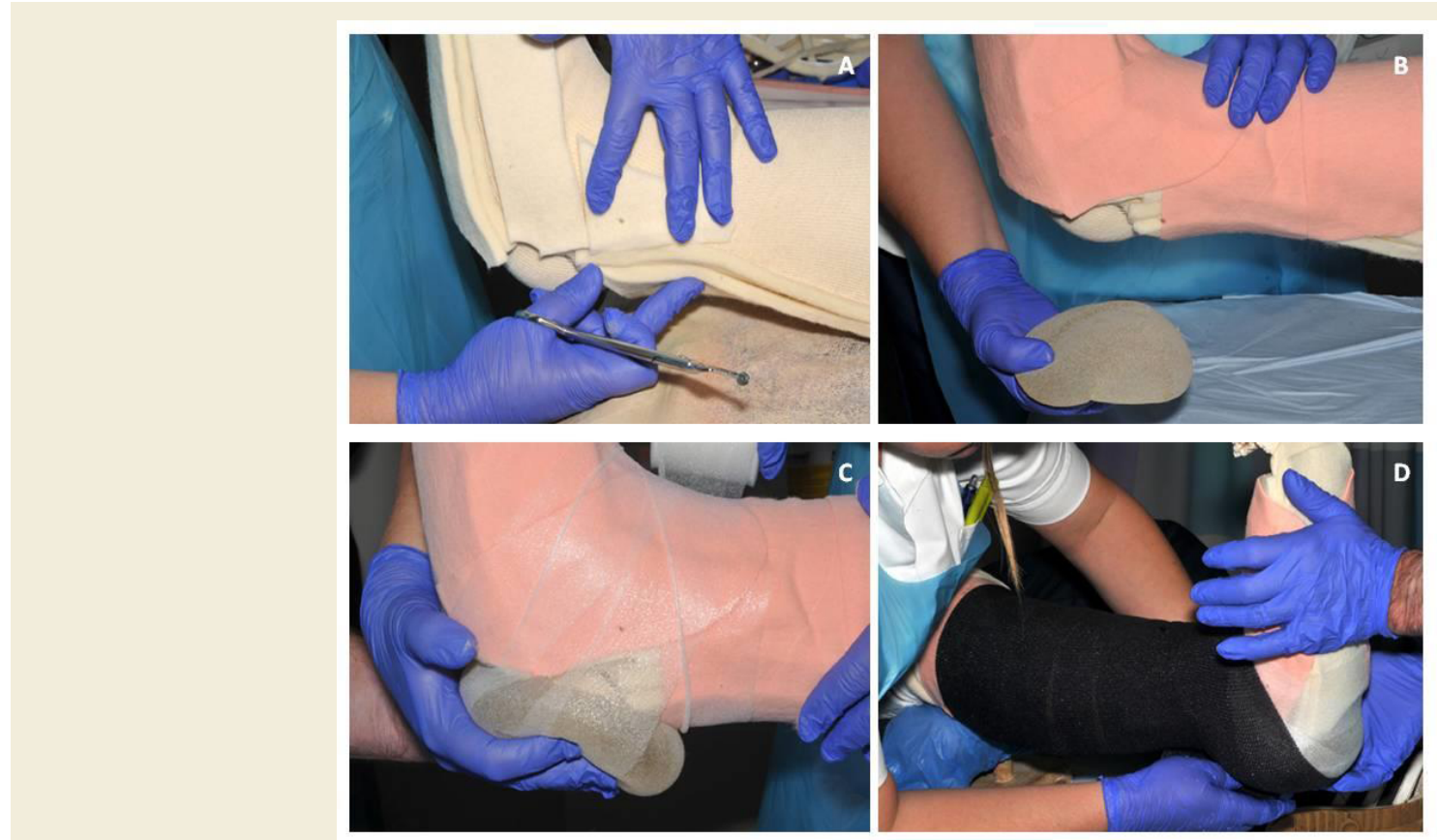

Figure 1: (A) Standard technique with double layer stockinet and under cast padding is applied. (B) This is covered with orthopaedic felt leaving the ulcerated area free. (C) A spare silicone breast implant is positioned at the heel. (D) Finally the plaster cast is completed; the whole construct can safely be made removable by splitting the cast. 
contact casting in this group of patients. Following adequate selection of cases, patients are provided with verbal and written information regarding this treatment strategy in order to improve compliance. We have developed an innovative technique to construct a cushion modified total-contact cast. A spare silicone breast implant is positioned at the heel in order to fill the defect at the ulcer site, while allowing the cast to conform to the shape of the limb and prevent padding layers from migrating into the area of defect. The implant is secured with pre-cast tape and the rest of the plaster completed in a standard fashion using synthetic cast (Figure 1A-1D). To our knowledge this technique has not been described previously. It has the advantage of being simple, safe and easily reproducible as it relies on off-the-shelf equipment. The off-loading properties of total contact casting have been enhanced with the use of the described cushion technique.

\section{Discussion}

A clear diagnostic algorithm for the diagnosis of neuropathic arthropathy and a multidisciplinary approach remain the most important facets of dealing with this condition [2]. Hastings et al recently described significant progression of the Charcot osteoarthropathy when compared with the non-diseased contralateral foot. Progression included deterioration of the architecture of the foot triggering further Charcot-effects such as micro-fractures, nonunion, dislocations, deformities, skin ulcers and a twelve-fold increase in the risk of amputation [3]. Following early diagnosis, the first line of treatment is immobilisation in a full contact cast in order to prevent the formation, or allow the healing, of ulcers and changes in bone.

Numerous techniques for off-loading Charcot foot are available, these offer varying rates of healing, ulcer size reduction and complications [1,4]. When employed inadequately, this treatment modality can lead to complications mainly exacerbation of pre-existing soft tissue loss and formation of new ulcers [5]. Our technique provides better pressure dispersion around the ulcer area, which contributes to a better ulcer healing rate. Preventing collapse of the soft dressing and cast material in the tissue defect area allows better conformity of the cast. This results in reduced mid foot pressure, reduced risk of further ulceration and better off-loading of the Charcot joints. A previous study has demonstrated optimisation of total contact casting with the addition of cushion modification [6]. The cushion modified total contact cast was shown to reduce the peak and mean pressure at the ulcer site by $70 \%$ and $60 \%$ respectively compared to reduction by $44 \%$ and $47 \%$ with conventional total casting $(\mathrm{P}<0.001)$. In our institution we had no incidence of complications in relation to silicone implant use. By improving on the existing technique we were able to reduce the frequency of cast changes, improve outcomes and reduce costs.

\section{References}

1. Chirstensen TM, Gade-Rasmussen B, Pedersen LW, Hommel E, Holstein $P E$, et al. (2012) Duration of off-loading and recurrence rate in charcot osteoarthropathy treated with less restrictive regimen with removable walker. J Diabetes Complications 26: 430-434.

2. Trieb K (2016) The Charcot foot: pathophysiology, diagnosis and classification. Bone Joint J 98-B: 1155-1159.

3. Hastings MK, Johnson JE, Strube MJ, Hildebolt CF, Bohnert KL, et al. (2013) Progression of foot deformity in Charcot neuropathic osteoarthropathy. J Bone Joint Surg Am 95: 1206-1213.

4. Faglia E, Caravaggi C, Clerici G, Sganzaroli A, Curci V, et al. (2010) Effectiveness of removable walker cast versus nonremovable fiberglass off-bearing cast in the healing of diabetic plantar foot ulcer: a randomized controlled trial. Diabetes Care 33: 1419-1423.

5. Guyton GP (2005) An analysis of iatrogenic complications from the total contact cast. Foot Ankle Int 26: 903-907.

6. Burns J, Begg L (2011) Optimising the offloading properties of the tota contact cast for plantar foot ulceration. Diabet Med 28: 179-185. 